\title{
Characterization of a novel loss-of-function variant in TDP2 in two adult patients with spinocerebellar ataxia autosomal recessive 23 (SCAR23)
}

\author{
Edoardo Errichiello ${ }^{1}$, Guido Zagnoli-Vieira ${ }^{2}$, Romana Rizzi ${ }^{3}$, Livia Garavelli ${ }^{3}$, Keith \\ Caldecott $^{2}$, and Orzetta Zuffardi ${ }^{1}$ \\ ${ }^{1}$ University of Pavia \\ ${ }^{2}$ University of Sussex \\ ${ }^{3}$ Azienda USL di Reggio Emilia
}

May 5, 2020

\begin{abstract}
TDP2 encodes a 5'-tyrosyl DNA phosphodiesterase that is required for the efficient repair of double-strand breaks (DSBs) induced by the abortive activity of TOP2. To date, only two homozygous variants in TDP2 have been reported in five patients from three unrelated pedigrees with spinocerebellar ataxia 23 (SCAR23). By whole-exome sequencing, we identified a novel TDP2 splice-site variant (c.636+3_636+6del) in two Italian siblings (aged 39 and 43) showing progressive ataxia, intellectual disability, speech delay, refractory seizures, and various physical anomalies. The variant caused exon 5 skipping with consequent nonsense-mediated mRNA decay and defective repair of TOP2-induced DSBs, as demonstrated by the functional assays performed on the patients' fibroblasts. Our findings further support the pathogenic role of biallelic loss-of-function variants of TDP2 in SCAR23 pathogenesis. Considering the adult age of our patients and their extensive follow-up, our study delineates in more detail the clinical phenotype related to the loss of TDP2 activity.
\end{abstract}

\section{KEYWORDS}

TDP2, topoisomerase II (TOP2), double-strand breaks (DSBs), spinocerebellar ataxia, intellectual disability, epilepsy, speech delay, whole-exome sequencing (WES)

The impairment of DNA repair systems and accumulation of DNA double strand breaks (DSBs) are involved in the pathogenesis of different neurodegenerative diseases, such as Alzheimer's disease and amyotrophic lateral sclerosis (Madabhushi et al., 2014). TDP2 (6p22.3) encodes the enzyme tyrosyl-DNA phosphodiesterase 2 that is required for efficient repair of topoisomerase II (TOP2)-induced DSBs by non-homologous end-joining (NHEJ) (Cortes Ledesma et al., 2009; Gómez-Herreros et al., 2013). In addition, TDP2 has been shown to be involved in neural development and maintenance by regulating brain-specific gene transcripts (Gómez-Herreros et al., 2014), and it is expressed throughout the brain of adult mice, especially in the dentate gyrus of the hippocampus and in the dopaminergic substantia nigraneurons, where it exerts a proapoptotic function by interacting with Parkinson's disease-associated DJ-1/PARK7 mutant proteins (Zucchelli et al., 2009).

Biallelic inactivating variants of $T D^{2} 2$ have been identified in five individuals with intellectual disability, seizures and ataxia, defining a new nosological entity known as spinocerebellar ataxia autosomal recessive 23 (SCAR23; OMIM \#616949) (Gómez-Herreros et al., 2014; Zagnoli-Vieira et al., 2018; Ciaccio et al., 2019). TOP2-induced DSBs have been implicated in genome instability and oncogenic chromosome translocation (Gómez-Herreros et al., 2017). On the other hand, the use of "TOP2 poisons" such as etoposide and 
doxorubicin has been suggested in clinical practice as adjuvant therapy to sensitize cancer cells toward TOP2 poisons (Kont et al., 2016).

We characterized a novel TDP2 variant in two adult siblings (aged 45 and 39 years), supporting the causative role of TDP2loss-of-function variants in SCAR23 and further delineating the related clinical phenotype. We also addressed the open question of whether TDP2 may be involved in susceptibility to other neurological conditions as well as cancer.

\section{Clinical phenotypes}

The probands are a 45-year-old male (III-38) and his 39-year-old sister (III-40), born to declared nonconsanguineous parents from the same small village of Southern Italy (Calabria). The second-born child, who died at 5 in a car accident, developed normal language skills (Figure 1a). The siblings were affected by global developmental delay (walking $>18$ months), ataxia, intellectual disability, speech delay (first words at 5-6 years), focal drug-resistant epileptic seizures (by the age of 15) and progressive gait deterioration. Furthermore, they showed several craniofacial dysmorphisms, hypertrichosis, abdominal distention, and flatfoot (Table 1). Brain MRI in the brother showed bilateral mesial temporal sclerosis (documented when the patient was 35 years old) and clinodactyly of the $5^{\text {th }}$ finger (Figure 1b). Both siblings achieved complete pubertal development. Metabolic tests (urinary amino and organic acids, lactate, pyruvate, ammonium), echocardiogram, audiometry, and first-level genetic screening (karyotyping, subtelomeric FISH) were normal. The proposita underwent bilateral cataract surgery at the age of 31 .

The family history was remarkable for other neuromuscular diseases in the maternal side of the pedigree, whereas no extensive information was available for paternal relatives. Two cousins (daughters of a mother's brother; III-1 and III-2) died of congenital muscular dystrophy at the age of 6 and 2 years, respectively; a 38-year-old cousin (son of a mother's brother; III-18) was affected by cognitive impairment since the age of 10, and developed hallucinations, social isolation, and aggressive mood at the age of 18, being diagnosed as paranoid schizophrenia (ICD-10); and a 28-year-old cousin (daughter of a mother's brother; III-28) was affected by epilepsy since the age of 3 . The mother of the probands developed a colorectal adenocarcinoma (TNM: pT3 N1b) when she was 51 years old.

Patient's data have been submitted to the ClinVar database with accession number SCV001147007. The study was conducted in accordance with the Declaration of Helsinki and national guidelines. Written informed consent was obtained from the patients' guardian for the publication of this study and any potentially identifying images.

\section{Genetic analysis and functional validation in the patients}

Whole-exome sequencing (WES; SureSelect Human All Exome V7) identified in both siblings a homozygous variant (NM_016614.3:c.636+3_636+6del) within the exon-intron boundary of TDP2 exon 5, removing 4 nucleotides near the splicing donor site (Figure S1). The variant, unreported in gnomAD and found in only one carrier individual in the Bravo/TOPMed dataset $(1 / 125,568,0.000796 \%)$, was at the heterozygous state in the healthy parents. Homozygosity mapping detected a large region of homozygosity (ROH) of $30 \mathrm{Mb}$ (chr6:10,398,474-40,431,707; GRCh38/hg38), shared by the affected siblings and spanning TDP2 (chr6:24,649,977-24,667,033).

Different bioinformatic tools predicted donor splice-site loss due to the c.636+3_636+6del variant. In agreement with the in-silicopredictions, fibroblasts' analysis revealed that the variant caused the skipping of TDP2 exon 5, leading to the insertion of a premature stop codon within six amino acid residues (p.His174GlufsTer6) and consequent nonsense-mediated mRNA decay (Figure 2a and Figure 2b). Accordingly, immunoblotting failed to detect TDP2 protein in the fibroblasts of both siblings, totally overlapping the expression pattern previously observed in the fibroblasts of the 850BR patient (Zagnoli-Vieira et al., 2018) harbouring the c. $425+1 \mathrm{G}>\mathrm{A}$ variant in TDP2 (Figure 2c). Furthermore, cells from affected individuals carrying either the c.636+3_636+6del or the c. $425+1 \mathrm{G}>$ A variants revealed defective repair of TOP2-induced DSBs relative to control cells, as measured by the persistence of $\gamma \mathrm{H} 2 \mathrm{AX}$ immunostaining, an indirect marker of DSBs, after 
treatment with the TOP2 poison etoposide (Figure 2d). According to the ACMG criteria (Richards et al., 2015), the variant was classified as pathogenic.

\section{Pedigree segregation and somatic analysis}

By Sanger sequencing, we detected the c.636+3_636+6del variant at the heterozygous state in one apparently healthy paternal uncle as well as in the cousin with schizophrenia (III-18). In this latter, the variant was inherited from the apparently unaffected father and was also present in the younger healthy brother (Figure 1a). WES analysis in III-18 excluded the presence of additional pathogenic variants in known schizophreniaassociated genes (Table S1) as well as copy number variations (CNVs). DNA from other family members was not available for the analysis. Considering the crucial role of TDP2 in NHEJ and maintenance of genome stability and the development of a colorectal cancer in the patients' mother, we were prompted to examine whether a second somatic hit in TDP2 may have occurred. However, any pathogenic coding variant was detected in the DNA obtained from the tumour sample.

\section{Discussion}

To date, five patients have been described to carry homozygous TDP2 variants. A homozygous splice-site variant in TDP2 (c. $425+1 \mathrm{G}>\mathrm{A})$ has been reported in three Irish siblings and in an additional patient from the United States who shared the same haplotype, suggesting a founder effect (Gómez-Herreros et al., 2014; Zagnoli-Vieira et al., 2018). Recently, a homozygous nonsense variant in the exon 3 of TDP2(c.400C $>\mathrm{T}$; p.Arg134Ter) has been detected in a 11-year-old Italian girl (Ciaccio et al., 2019).

In this study we identified a novel homozygous spice-site variant disrupting the correct splicing of TDP2 exon 5. By using immunofluorescent detection of $\gamma \mathrm{H} 2 \mathrm{AX}$ as an indirect measure of DSBs, we demonstrated that the novel TDP2 c.636+3_636+6del splice-site variant (as the c.425+1G>A) profoundly impacts on the nuclear DSB repair after treatment with etoposide. Although the functional effect of the c.400C $>\mathrm{T}$ (p.Arg134Ter) variant was not established, it is expected to lead to nonsense-mediated mRNA decay, as in the case of the c. $425+1 \mathrm{G}>\mathrm{A}$ and the c.636+3_636+6del splice-site variants.

The five previous and our current patients share several clinical features, mainly craniofacial and neurological (Table 1). Particularly, 5 out of 7 patients with TDP2 inactivation showed speech impairment, which may be considered a recurrent feature in SCAR23 along with drug-resistant epileptic seizures and intellectual disability - shared by all described cases, progressive ataxia (6/7), and craniofacial dysmorphisms $(6 / 7)$ (Table 1). We previously observed neutropenia in a 6 -year-old child carrying the c.425+1G $>$ A variant (Zagnoli-Vieira et al., 2018). Interestingly, TDP2 is highly expressed in the hemolymphoid system and interacts with CD40, a member of the tumour necrosis factor (TNF) receptor family that plays a critical role in several immunological processes (Pype et al., 2000), and mutations of $C D 40$ gene have been associated to an autosomal recessive form of immunodeficiency with hyper IgM and neutropenia (OMIM \#606843; Ferrari et al., 2001). Nevertheless, haematological manifestations have not been reported in other patients and do not seem to be a pathognomonic signature for SCAR23, at least based on current observations in patients.

Our patients manifested eye anomalies, particularly early-onset bilateral cataracts in the proposita, a feature never described in SCAR23. The extended in silico panel for cataract (Table S2) was negative, a finding against a potential multilocus inheritance. Interestingly, the exon 5 of TDP2 is predicted to contain a 21-bp transcription factor binding site for PAX6 (chr6:24,654,475-24,654,495, GRCh38/hg38; V\$PAX6_01, Transfac Matrix Database v.7.0), whose deficiency is associated with distinct ocular phenotypes including cataract (OMIM \#106210). Although more functional studies are needed to support this hypothesis, TDP2 could be involved in the gene regulatory circuitry of PAX6/SOX2 that orchestrates lens development and neurogenesis.

TDP2 has been recognized as a key player for neural homeostasis because of its role in preventing DNA damage led by abortive TOP2 activity. Curiously, biallelic inactivation of TDP1 causes spinocerebellar ataxia with axonal neuropathy (SCAN1; OMIM \#607250) (Takashima et al., 2002), further supporting the central role of topoisomerases and their partner tyrosyl-DNA phosphodiesterases in neurodevelopment. Moreover, 
Stoll et al. (2013) found a significant enrichment of a rare 240-kb chromosome 22q11.22 microdeletion spanning TOP3B (encoding the DNA topoisomerase III $\beta$ ) in Finnish patients affected by schizophrenia and cognitive impairment, whereas mutation of TOP1 (encoding the DNA topoisomerase I) has been implicated in autism spectrum disorders (King et al., 2013; McKinnon, 2016). Interestingly, a small inherited duplication in 6p22.3 (37.89 kb) joining the first noncoding exon of KIAA0319 with the 3' end of the neighbouring gene TDP2 (exons 4-7) was identified in an Irish patient with autism spectrum disorder (ASD) (Holt et al., 2012). In Decipher, another inherited heterozygous duplication of KIAA0319 - TDP2 with similar size (33.43 kb) was detected in a female patient (\#281899) with delayed speech and infantile spasms. Although several studies implicated KIAA0319 in dyslexia susceptibility (Francks et al., 2004; Cope et al., 2005; Harold et al., 2006; Paracchini et al., 2006; Dennis et al., 2009; Eicher et al., 2014), a condition frequently associated with ASD, we cannot rule out the involvement of TDP2 , a gene that "keeps the brain healthy" (McKinnon, 2014), as susceptibility factor in the development of schizophrenia when combined with other genetic and environmental factors. In this regard, we detected the c.636+3_636+6del variant at the heterozygous state in the DNA of the probands' cousin suffering from schizophrenia. Although the variant was inherited from the father, with no apparent neurobehavioral abnormalities, a possible involvement of TDP2 may not be completely ruled out and merits further investigations.

Apart from their role in normal brain function, topoisomerases, when altered and mis-expressed, may play a critical role in chromosome instability (CIN), a hallmark of carcinogenesis (Liu et al., 2019). Clinically, a large arsenal of topoisomerase inhibitors has been used in personalized cancer therapy to suppress DNA replication of tumour cells (Chen et al., 2015). Several studies showed that TDP2-dependent repair of DSBs protects cells from genome instability and chromosome translocations (Gómez-Herreros et al., 2013, 2017; Canela et al., 2019). Moreover, TDP2 has been suggested as a promising target for anticancer therapy, further underlying its possible role in carcinogenesis. According to the COSMIC database, 94 different variants in TDP2 , mostly missense substitutions (76.7\%), have been detected in 103 out of 35,902 tumour samples $(0.28 \%)$ tested from different anatomical regions, with an overall enrichment for the large intestine $(28 / 2331$, $1.2 \%$ ). These data raise the question of whether SCAR23 patients and TDP2 mutation carriers may be more prone to develop tumours and need specific oncological follow-up. Although more observations are needed, we excluded the presence of a second coding hit in the tumour sample of the patients' mother, weakening a possible involvement of TDP2 as a tumour suppressor gene in colorectal carcinogenesis. In this regard, it should be also considered that other redundant mechanisms are available to repair TOP2-induced DSBs, such as the Mre11-dependent homologous recombination (Hoa et al., 2016).

In summary, our results strongly suggest that biallelic loss-of-function variants in TDP2 are associated with SCAR23. Whether the accumulation of other classes of variants, such as missense substitutions in crucial TDP2 functional domains, especially the C-terminal exonuclease-endonuclease-phosphatase (EEP) catalytic domain (aa 117-351), might be associated with distinct phenotypes or degrees of severity needs further investigations.

\section{CONFLICT OF INTERESTS}

The authors have no conflicting interests to declare.

DATA AVAILABILITY STATEMENT The data that supports the findings of this study are available in the supplementary material of this article. Any other data will be made available on request from the corresponding author.

\section{REFERENCES}

Canela, A., Maman, Y., Huang, S.N., Wutz, G., Tang, W., Zagnoli-Vieira, G., .. Nussenzweig, A. (2019). Topoisomerase II-Induced Chromosome Breakage and Translocation Is Determined by Chromosome Architecture and Transcriptional Activity. Molecular Cell , 75(2):252-266.e8.

Chen, T., Sun, Y., Ji, P., Kopetz, S., Zhang, W. (2015). Topoisomerase II $\alpha$ in chromosome instability and personalized cancer therapy. Oncogene, 34 (31):4019-31. 
Ciaccio, C., Castello, R., Esposito, S., Pinelli, M., Nigro, V., Casari, G., Chiapparini, L., Pantaleoni, C; TUDP Study Group, D'Arrigo, S. (2019). Consolidating the Role of TDP2 Mutations in Recessive Spinocerebellar Ataxia Associated with Pediatric Onset Drug Resistant Epilepsy and Intellectual Disability (SCAR23). Cerebellum, 18 (5):972-975.

Cope, N., Harold, D., Hill, G., Moskvina, V., Stevenson, J., Holmans, P., Owen, M. J., O'Donovan, M. C., Williams, J. (2005). Strong evidence that KIAA0319 on chromosome $6 \mathrm{p}$ is a susceptibility gene for developmental dyslexia. The American Journal of Human Genetics ,76 (4):581-91.

Cortes Ledesma, F., El Khamisy, S. F., Zuma, M. C., Osborn, K., Caldecott, K. W. (2009). A human 5'-tyrosyl DNA phosphodiesterase that repairs topoisomerase-mediated DNA damage. Nature,461 (7264), $674-8$.

Dennis, M. Y., Paracchini, S., Scerri, T. S., Prokunina-Olsson, L., Knight, J. C., Wade-Martins, R., Coggill, P., Beck, S., Green, E. D., Monaco, A. P. (2009). A common variant associated with dyslexia reduces expression of the KIAA0319 gene. PLoS Genetics, 5 (3):e1000436.

Eicher, J.D., Powers, N.R., Miller, L.L., Mueller, K.L., Mascheretti, S., Marino, C., .. Gruen, J.R. (2014). Characterization of the DYX2 locus on chromosome 6p22 with reading disability, language impairment, and IQ. Human Genetics , 133 (7):869-81.

Ferrari, S., Giliani, S., Insalaco, A., Al-Ghonaium, A., Soresina, A.R., Loubser, M., .. Plebani, A. (2001). Mutations of CD40 gene cause an autosomal recessive form of immunodeficiency with hyper IgM.Proceedings of the National Academy of Sciences USA ,98 (22):12614-9.

Francks, C., Paracchini, S., Smith, S. D., Richardson, A. J., Scerri, T. S., Cardon, L. R. (2004). A 77-kilobase region of chromosome $6 \mathrm{p} 22.2$ is associated with dyslexia in families from the United Kingdom and from the United States. The American Journal of Human Genetics, 75 (6):1046-58.

Gómez-Herreros, F., Romero-Granados, R., Zeng, Z., Alvarez-Quilón, A., Quintero, C., Ju, L., ... CortésLedesma, F. (2013). TDP2-dependent non-homologous end-joining protects against topoisomerase II-induced DNA breaks and genome instability in cells and in vivo. PLoS Genetics, 9 (3):e1003226.

Gómez-Herreros, F., Schuurs-Hoeijmakers, J. H., McCormack, M., Greally, M. T., Rulten, S., RomeroGranados, R., ... Caldecott, K. W. (2014). TDP2 protects transcription from abortive topoisomerase activity and is required for normal neural function. Nature Genetics ,46 (5):516-21.

Gómez-Herreros, F., Zagnoli-Vieira, G., Ntai, I., Martínez-Macías, M. I., Anderson, R. M., Herrero-Ruíz, A., Caldecott, K. W. (2017). TDP2 suppresses chromosomal translocations induced by DNA topoisomerase II during gene transcription. Nature Communications , 8 (1):233.

Harold, D., Paracchini, S., Scerri, T., Dennis, M., Cope, N., Hill, G., .. Monaco, A.P. (2006). Further evidence that the KIAA0319 gene confers susceptibility to developmental dyslexia. Molecular Psychiatry, 11 (12):1085-91.

Hoa, N. N., Shimizu, T., Zhou, Z. W., Wang, Z. Q., Deshpande, R. A., Paull, T. T., .. Sasanuma, H. (2016). Mre11 Is Essential for the Removal of Lethal Topoisomerase 2 Covalent Cleavage Complexes.Molecular Cell, 64 (3):580-592.

Holt, R., Sykes, N. H., Conceição, I. C., Cazier, J. B., Anney, R. J., Oliveira, G., Gallagher, L., Vicente, A., Monaco A. P., Pagnamenta, A. T. (2012). CNVs leading to fusion transcripts in individuals with autism spectrum disorder. European Journal of Human Genetics, 20 (11):1141-7.

King, I. F., Yandava, C. N., Mabb, A. M., Hsiao, J. S., Huang, H. S., Pearson, B. L., . . Zylka, M. J. (2013). Topoisomerases facilitate transcription of long genes linked to autism. Nature, 501 (7465):58-62.

Kont, Y.S., Dutta, A., Mallisetty, A., Mathew, J., Minas, T., Kraus, C., ... Adhikari, S. (2016). Depletion of tyrosyl DNA phosphodiesterase 2 activity enhances etoposide-mediated double-strand break formation and 
cell killing. DNA Repair, $43: 38-47$.

Liu, T., Zhang, H., Yi, S., Gu, L., Zhou, M. (2019). Mutual regulation of MDM4 and TOP2A in cancer cell proliferation. Molecular Oncology, 13 (5):1047-1058.

Madabhushi, R., Pan, L., Tsai, L. H. (2014). DNA damage and its links to neurodegeneration. Neuron , 83 (2), 266-282.

McKinnon, P. J. (2014). TDP2 keeps the brain healthy. Nature Genetics, 46 (5):419-21.

McKinnon, P. J. (2016). Topoisomerases and the regulation of neural function. Nature Reviews Neuroscience, 17 (11):673-679.

Paracchini, S., Thomas, A., Castro, S., Lai, C., Paramasivam, M., Wang, Y., ... Monaco, A. P. (2006). The chromosome 6p22 haplotype associated with dyslexia reduces the expression of KIAA0319, a novel gene involved in neuronal migration. Human Molecular Genetics, 15 (10):1659-66.

Pype, S., Declercq, W., Ibrahimi, A., Michiels, C., Van Rietschoten, J. G., Dewulf, N., de Boer, M., Vandenabeele, P., Huylebroeck, D., Remacle, J. E. (2000). TTRAP, a novel protein that associates with CD40, tumor necrosis factor (TNF) receptor-75 and TNF receptor-associated factors (TRAFs), and that inhibits nuclear factor-kappa B activation. The Journal of Biological Chemistry, 275 (24):18586-93.

Richards, S., Aziz, N., Bale, S., Bick, D., Das, S., Gastier-Foster, J., .. Rehm, H.L. (2015). Standards and guidelines for the interpretation of sequence variants: a joint consensus recommendation of the American College of Medical Genetics and Genomics and the Association for Molecular Pathology. Genetics in Medicine ,17 (5):405-24.

Stoll, G., Pietiläinen, O. P. H., Linder, B., Suvisaari, J., Brosi, C., Hennah, W. (2013). Deletion of TOP3ß, a component of FMRP-containing mRNPs, contributes to neurodevelopmental disorders. Nature Neuroscience, 16 (9):1228-1237.

Takashima, H., Boerkoel, C. F., John, J., Saifi, G. M., Salih, M. A., Armstrong, D., .. Lupski, J. R. (2002). Mutation of TDP1, encoding a topoisomerase I-dependent DNA damage repair enzyme, in spinocerebellar ataxia with axonal neuropathy. Nature Genetics, 32 (2):267-72.

Zagnoli-Vieira, G., Bruni, F., Thompson, K., He, L., Walker, S., de Brouwer, A. P. M., Taylor, R., Niyazov, D., Caldecott, K. W. (2018). Confirming TDP2 mutation in spinocerebellar ataxia autosomal recessive 23 (SCAR23). Neurology Genetics , 4 (4):e262.

Zucchelli, S., Vilotti, S., Calligaris, R., Lavina, Z. S., Biagioli, M., Foti, R., .. Gustincich, S. (2009). Aggresome-forming TTRAP mediates pro-apoptotic properties of Parkinson's disease-associated DJ-1 missense mutations. Cell Death and Differentiation, 16 (3):428-38.

\section{FIGURE LEGENDS}

FIGURE 1. (a) Family pedigree showing a miscellany of neurological phenotypes in the last generation of the family. +/+ homozygous wild-type; +/- heterozygous carrier; -/- homozygous mutant for the TDP2 c.636+3_636+6del variant. (b) Clinical features of the probands (III-38 and III-40), showing highly overlapping facial dysmorphisms, large hands, clinodactyly of the $5^{\text {th }}$ finger, and flatfoot.

FIGURE 2 . Functional analysis on the probands' fibroblasts. (a) RT-PCR shows exon 5 skipping at the RNA level in the probands compared to control pools; NTC: no template control. (b) Sequencing analysis of the exon 5 skipped band in the RT-PCR. The junction between exon 4 and exon 6 creates a frame shift leading to a premature termination codon within six amino acid residues. (c) TDP2 immunoblotting showing no detectable TDP2 expression in either Italian (novel mutation) or US patients (previous mutations); 1BR: control human fibroblast cell line; 850BR: TDP2-mutated patient primary human fibroblasts; III-38, III-40: probands' fibroblasts; YJR1504: primary fibroblast control cell line. (d) Repair kinetics of yH2AX foci after 
$25 \mu \mathrm{M}$ etoposide treatment showing decreased repair of TOP2-induced DSBs in affected cells (probands and 850BR) compared to control cell line (1BR). Data are the mean ( \pm s.e.m.) of four independent experiments.

FIGURE S1. WES analysis and segregation in the family. WES results visualized on the Integrative Genomics Viewer (IGV) in the probands and parents (top left panel). NGS findings were confirmed by Sanger sequencing (top right panel), which was also used for segregation analysis in other family members (bottom panel).

\section{Hosted file}

Figure 1.tif available at https://authorea.com/users/301643/articles/431493-characterizationof-a-novel-loss-of-function-variant-in-tdp2-in-two-adult-patients-with-spinocerebellarataxia-autosomal-recessive-23-scar23

\section{Hosted file}

Figure 2.tif available at https://authorea.com/users/301643/articles/431493-characterizationof-a-novel-loss-of-function-variant-in-tdp2-in-two-adult-patients-with-spinocerebellarataxia-autosomal-recessive-23-scar23 